\title{
Comparação da ativação mioelétrica do glúteo máximo e bíceps femoral entre os agachamentos paralelo e com passada à frente
}

CDD. 20.ed. 616.7

796.073

\author{
Gustavo LEPORACE* \\ Glauber Ribeiro PEREIRA* \\ Luis Carlos Nascimento da COSTA* \\ Luiz Cláudio TEIXEIRA** \\ Luiz Alberto BATISTA* \\ *Universidade do Esta- \\ do do Rio de Janeiro. \\ **Universidade Estácio \\ de Sá.
}

\section{Resumo}

0 objetivo desse estudo foi comparar a atividade mioelétrica (EMG) do glúteo máximo e bíceps femoral entre o agachamento com passada à frente e com pés paralelos. Sete indivíduos realizaram oito repetições do agachamento paralelo (AP) e com passada à frente (AF) deslocando sobrecarga relativa a 50\% da massa corporal. 0 EMG do glúteo máximo e bíceps femoral foi capturado, filtrado por um filtro passa-banda Butterworth de $4^{\mathrm{a}}$ ordem $(20-400 \mathrm{~Hz})$ e obtido valores RMS. Utilizou-se o teste de Wilcoxon para comparar o EMG normalizado entre os dois exercícios. Tanto o bíceps femoral $(p=0,041)$ quanto o glúteo máximo $(p=0,0059)$ apresentaram maior ativação no $\operatorname{AF}(30,9 \%$ para glúteo; $42,6 \%$ para bíceps) em comparação ao $\operatorname{AP}(21,2 \%$ para glúteo; $24,7 \%$ para bíceps). Apesar da ativação moderada durante a execução dos exercícios, girando em torno de $25 \%$ a $45 \%$, a resposta foi maior, para os participantes do estudo, no AF.

UnITERMOS: Eletromiografia; Treinamento de resistência; Quadril.

\section{Introdução}

É comum que profissionais que atuam no campo do treinamento desportivo selecionem determinados exercícios físicos por considerá-los mais eficazes que outros, no que tange à capacidade de estimular respostas musculares. Porém, parecem ser recorrentes os casos em que o esteio de conhecimentos adotado na fundamentação desse juízo não seja massivamente oriundo de fontes cientificas, o que encurte um significativo grau de incerteza no processo. Nesse sentido, no que tange à necessidade de distinguir as modalidades de agachamento, análises biomecânicas e cinesiológicas constituem estratégias apropriadas à caracterização dos exercícios, uma vez que elas possibilitam precisar as diferenças nas configurações de movimento e estimar os efeitos de estimulação sobre os músculos.

Entre as estratégias disponíveis para avaliar o grau de eficácia de exercícios no treinamento de força, a técnica de eletromiografia de superfície (EMG) é uma das mais utilizadas por possibilitar análises acuradas, tanto qualitativas quanto quantitativas, da atividade mioelétrica manifesta durante o ato da exercitação (DE LuCA, 2007). Decorrentes disso, diferentes estudos foram realizados com o propósito de se examinar a atividade de músculos dos membros inferiores durante a execução de exercícios, tais como o agachamento (Caterisano, Moss, Pellinger, Woodruff, Lewis, Booth \& Khadra, 2002; DA Silva, Brentano, Cadore, De Almeida \& Kruel, 2008; Pereira, Leporace, Chagas, Furtado, PraXEDEs \& BATISTA, 2010). Com base nos resultados desses estudos é razoável inferir que o recrutamento muscular é influenciado por distintos fatores, tais como: amplitude de movimento, posição articular e intensidade do exercício (CATERISANO et al., 2002; Da SiLVA et al., 2008; Pereira et al., 2010).

Nessa mesma linha de investigação um aspecto discutido na literatura de treinamento de força diz 
respeito à questão de qual tipo de exercício melhor estimula a resposta da musculatura glútea e posteriores de coxa. Escamilla, Fleisig, Zheng, Lander, Barrentine, Andrewa, Bergemann e Moorman (2001) constataram que exercícios de agachamento destacam-se na capacidade de estimular os referidos músculos, quando comparados a outros que também o fazem. No entanto, não se sabe qual, dentre os diferentes tipos de agachamento, promove uma maior resposta do glúteo máximo e posteriores de coxa.

Dentre os distintos tipos utilizados na exercitação da referida musculatura encontra-se o agachamento com passada à frente, o qual pode ser descrito como uma variação do agachamento tradicional, que se caracteriza pelo fato do executante realizá-lo com um dos membros

\section{Método}

\section{Amostra}

Cinco homens $(84 \pm 14,6 \mathrm{~kg}, 1,72 \pm 0,07 \mathrm{~m}$, $26 \pm 4,2$ anos) e 2 mulheres $(54,5 \pm 9,1 \mathrm{~kg}, 1,59$ $\pm 0,05 \mathrm{~m}, 24,5 \pm 2,1$ anos) voluntariaram-se para participar do estudo após assinatura do Termo de Consentimento Livre e Esclarecido aprovado, assim como foram todos os procedimentos adotados na realização desse projeto, pelo Comitê de Ética em Pesquisa da Universidade do Estado do Rio de Janeiro, sob o parecer no 032.3.2009.

Para serem incluídos na pesquisa os indivíduos, no momento da coleta de dados, deveriam apresentar vivência na realização do exercício agachamento de pelo menos um ano e estar engajados em programas de treinamento contra resistência pelo menos três vezes por semana. Os participantes que apresentavam até o momento de realização do experimento, sinais ou relataram sintomas sugestivos de lesão osteomioarticular foram excluídos do estudo.

\section{Procedimentos}

Os indivíduos realizaram os exercícios Agachamento Paralelo (AP) e Agachamento com Passada à frente (AF) (FIGURA 1) com uma sobrecarga estipulada para a realização dos exercícios de 50\% da massa corporal total individual, sendo realizadas oito repetiçōes. O intervalo entre a série de cada exercício foi de 10 minutos para evitar que a fadiga interferisse nos resultados (Pereira et al., 2010). A ordem de realização dos exercícios foi randomizada. inferiores posicionado à frente, no plano sagital, e lateralmente ao outro, no plano frontal, assim como por apresentar uma cinética diferente da manifesta no agachamento padrão, com os pés paralelos (EsCAMILLA, Zheng, Macleod, Edwards, Hreljac, Fleisig, Wilk, MOORMAN, IMAMURA \& ANDREWS, 2008).

O objetivo neste estudo foi comparar a atividade mioelétrica do glúteo máximo e bíceps femoral entre o agachamento com passada à frente e o agachamento paralelo. A hipótese experimental foi de que, sendo utilizado um mesmo valor de sobrecarga absoluta, considerando a comparação pareada dos executantes, o agachamento passada, quando comparado ao agachamento paralelo, induz a maior ativação elétrica nos dois músculos.

Em ambos os agachamentos o executante iniciou o ciclo de movimento com o joelho em completa extensão e realizou um deslocamento angular de $90^{\circ}$ completando a fase descendente. Para assegurar que a amplitude de movimento (ADM) almejada fosse alcançada em cada meio ciclo de execução, foi utilizado um mecanismo sensibilizador posicionado posteriormente ao sujeito, que foi calibrado, por meio de comparação direta com um goniômetro manual (CARCI, Brasil). Dessa forma, foi possível controlar a ADM durante a execução utilizando, para efeito de "feedback", tanto informação tátil como verbal. O dispositivo sensibilizador foi ajustado para cada indivíduo de forma que a articulação do joelho não ultrapassasse os previstos $90^{\circ}$ de flexão. Um metrônomo Qwiktime, ajustado em $1 \mathrm{~Hz}$, determinando uma velocidade angular média de $45 \%$, foi utilizado no controle da frequência e do ritmo de execução.

A atividade mioelétrica (EMG) foi capturada a uma frequência de amostragem de $2 \mathrm{kHz}$ (EMG 100B, BIOPAC Systems Inc., Santa Bárbara, CA, USA), com amplificação bipolar diferencial (impedância de entrada $=2 \mathrm{M} \Omega$, taxa de rejeição do modo comum $>110 \mathrm{db}$, ganho $=1000)$, convertido analógico-digital (12 bit, MP100WSW BIOPAC Systems Inc) e armazenada em um computador pessoal para análise posterior.

Antes do posicionamento dos eletrodos, foi realizada a tricotomia e limpeza da pele com álcool isopropil, visando reduzir sua impedância (DELUCA, 1997). Para reduzir a influencia dos artefatos de movimento na atividade mioelétrica os cabos dos 
eletrodos foram fixados à pele do executante por meio de fita adesiva hipoalergênica (3M Ltda, Brasil).

Eletrodos de Ag/AgCl (KOBME Bio Protec Corp, Korea) foram, então, fixados na região do músculo glúteo máximo e bíceps femoral em paralelo com as fibras musculares, sendo utilizada uma distância intereletrodos de $2 \mathrm{~cm}$, segundo estratégia proposta por Cram, Kasman e Holtz (1998). O posicionamento dos eletrodos foi confirmado através de testes de contração isométrica voluntária máxima associada à palpação manual. Em ambos os exercícios, os eletrodos foram posicionados no membro inferior direito, sendo que no AF esse permaneceu à frente do esquerdo.

Os sinais eletromiográficos foram filtrados no sentido direto e reverso, para evitar distorções de fase, por um filtro Butterworth de $4^{\mathrm{a}}$ ordem com frequências de corte inferior e superior de $20 \mathrm{~Hz}$ e 400 $\mathrm{Hz}$, respectivamente, sendo calculado um valor de Raiz Média Quadrática parcial (Root Mean Square, RMSp), a partir do sinal mioelétrico, amostrado no intervalo de $60^{\circ}$ a $90^{\circ}$ de flexão do joelho na fase concêntrica da exercitação. Com o propósito de reduzir a significância da interveniência da fadiga muscular e da adequação ao padrão de movimento na configuração do sinal mioelétrico, o valor de RMS resultante (RMSr), efetivamente utilizado no exame das diferenças, foi obtido por meio do cálculo da média aritmética dos valores de RMSp dos quatro ciclos centrais de toda a exercitação, para cada sujeito. Este valor foi normalizado usando como referência a maior média do RMS obtido em duas contraçōes isométricas voluntárias máximas
(CIVM) de seis segundos, realizadas com resistência manual (Pereira et al., 2010). Para o glúteo máximo realizou-se extensão de quadril isométrica resistida em posição de decúbito ventral no solo. Para o bíceps femoral realizou-se uma flexão de joelho isométrica resistida com o indivíduo sentado e o joelho flexionado a $45^{\circ}$. O processamento dos sinais obtidos nas CIVMs foi semelhante ao descrito acima.

Como estratégia para estabelecer o sincronismo entre o eletromiograma e o decurso do deslocamento angular foi utilizado o sinal de um goniômetro flexível (TSD 130B, BIOPAC Systems Inc, Santa Bárbara, CA) fixado no eixo de movimento do joelho. A captura dos sinais da eletrogoniometria foi realizada a $2 \mathrm{kHz}$ e a calibração do goniômetro foi feita por meio de amostras de registros coletados a $0^{\circ}$ e $90^{\circ}$ de flexão do joelho, de acordo com as recomendações do manual do MP100 (BIOPAC Systems Inc, Santa Barbara, CA). Todos os dados mioelétricos foram processados através de rotinas desenvolvidas no "software” MatLab (versão 7.0.4, The MathWorks, USA).

\section{Análise estatística}

O tratamento estatístico dos dados foi realizado no "software" GraphPad Prim versão 5.0 (GraphPad software, EUA). Foi utilizado o Wilcoxon Rank test para verificar a presença de diferenças estatisticamente significantes entre os valores RMS normalizados provenientes dos exercícios AP e AF, sendo considerado um nível de significância de 5\%.

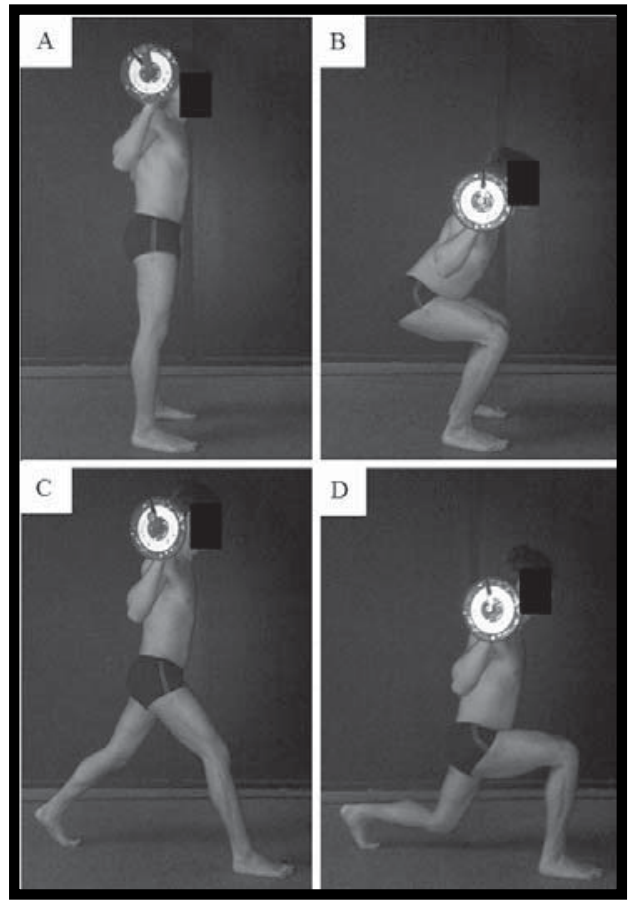

$A=$ Posição inicial do agachamento com pés paralelos (AP); $B=$ Posição final do AP $C=$ Posição inicial do agachamento com passada à frente (AF); $\mathrm{D}=$ Posição final do AF.

FIGURA 1 - Exercícios realizados no estudo. 


\section{Resultados}

Tanto o bíceps femoral $(\mathrm{p}=0,041)$ assim como o glúteo máximo $(\mathrm{p}=0,0059)$ apresentou maior ativação no $\mathrm{AF}$ em comparação ao $\mathrm{AP}$. $\mathrm{A}$ ativação das musculaturas foi mediana durante a

${ }^{*} p<0,05$;

${ }^{\star \star} p<0,01$ em relação ao AP. execução dos exercícios, com respeito à amplitude entre $60^{\circ}$ e $90^{\circ}$ de flexão do joelho, girando em torno de $25 \%$ a $45 \%$ da ativação relativa à CIVM (FIGURA 2).
Bíceps Femoral

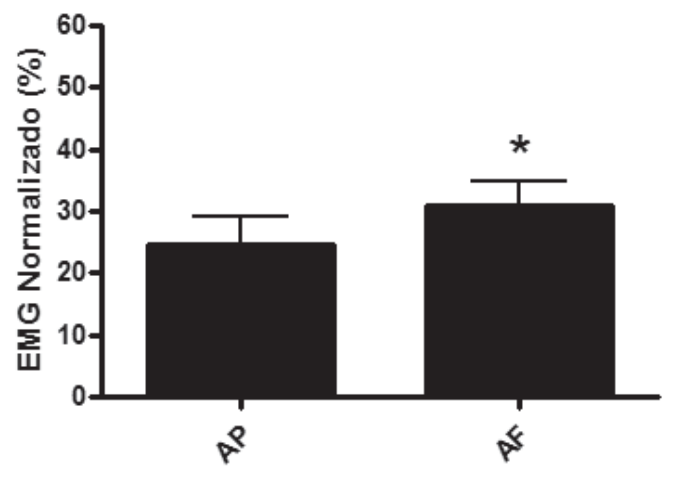

Glúteo Máximo

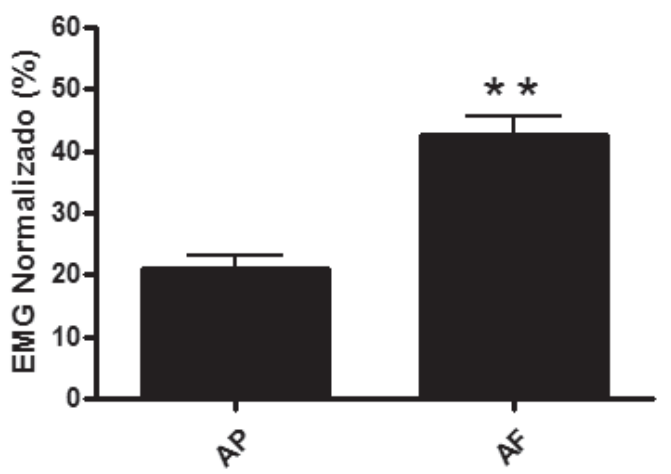

FIGURA 2 - Atividade mioelétrica do Bíceps Femoral e Glúteo Máximo no Agachamento Paralelo (AP) e Agachamento com Passada à Frente (AF).

\section{Discussão}

O objetivo do presente estudo foi comparar a atividade mioelétrica do glúteo máximo e do bíceps femoral entre os exercícios de AP e AF. Constatamos um percentual de ativação moderado nos dois exercícios (FIGURA 2), variando entre 25\% a 45\% da CIVM. Quanto a isso há que se registrar que as sobrecargas variaram entre 24 e $51 \mathrm{~kg}$, correspondendo, provavelmente, a um baixo percentual relativo do valor de $1 \mathrm{RM}$, para os indivíduos participantes (FLECK, 1999). Essa proposição está de acordo com a manifestação dos próprios participantes que relataram uma percepção subjetiva de esforço entre leve e moderado quando da execução de ambos os exercícios.

FLECK (1999) reportou que indivíduos considerados treinados realizam em média 1 RM do agachamento paralelo com sobrecarga por volta de $120 \%$ a $150 \%$ da massa corporal. CHAGAS, BARBOSA e LIMA (2005) relataram que homens e mulheres realizaram em média, respectivamente, 7,6 e 7,7 repetições no exercício de "Leg Press" a 80\% de 1 RM. Por outro lado, a mesma amostra realizou, respectivamente, 20,8 e 20,7 repetições do mesmo exercício a $40 \%$ de 1 RM. Apesar das diferenças entre o "Leg Press" e o agachamento, tais exercícios apresentam algumas características semelhantes como articulações mobilizadas, plano de movimento e grupamentos musculares ativados, o que possibilita certo grau de inferência dos resultados obtidos pelos pesquisadores. Neste sentido, é razoável pressupor que os indivíduos realizaram com relativa facilidade oito repetiçóes do agachamento com a sobrecarga absoluta de 50\% da massa corporal utilizada neste trabalho, uma vez que a carga para $1 \mathrm{RM}$, para o mesmo número de repetições, provavelmente seria maior.

Sendo assim, é possível sugerir que um aumento do valor da carga resistiva promovesse, de forma associada, um aumento, mesmo que não linear, da atividade muscular (DE LUCA, 1997), e propiciasse o alcance das magnitudes de respostas musculares necessárias à obtenção de efeitos de treinamento diversos, como, por exemplo, o aumento da capacidade de geração de força e de hipertrofia muscular. No entanto, a possibilidade de se obter, efetivamente, os referidos efeitos de treinamento devem ser estudados em outra oportunidade uma vez não ter sido objeto da presente investigação.

Apesar do moderado percentual de ativação encontrado, a hipótese de estudo, de que o AF promoveria uma maior ativação nos músculos examinados, em comparação ao AP, foi confirmada pelos resultados. Esses achados estão em acordo com os obtidos por 
McCaw e Melrose (1999) e Pereira et al. (2010), os quais, embora tenham estudado a atividade de músculos diferentes dos aqui investigados, também preocuparam-se em verificar em que medida distintas disposiçôes corporais se associam à diferentes respostas mioelétricas. Em ambos os casos os pesquisadores identificaram que adutores de quadril aumentam sua atividade mioelétrica, quando sujeitos a uma situação de geometria articular que, provavelmente, induz o pré-estiramento muscular e apontaram esse fenômeno como causa provável do aumento na atividade muscular, para o que basearam-se no pressuposto de que músculos que se encontram com certo grau de estiramento antes de iniciar a contração têm vantagem mecânica na geração de força em relação a músculos que se encontram com o comprimento de repouso, o que, consequentemente, resulta em uma maior capacidade de gerar tensão durante a realização de exercícios (FLECK \& KRAEMER, 1999).

A disposição corporal adotada no AF, com um membro inferior adiante do outro, também resulta em uma geometria articular de membros inferiores que, do ponto de vista cinesiológico, tende a propiciar uma melhor condição de pré-estiramento tanto da musculatura glútea quanto dos posteriores de coxa, sendo esperado que nessa condição as atividades mioelétricas se manifestem em maior magnitude (Dwyer, Boudreau, Mattacola, Uhl \& LatTerman, 2010), manifestação essa observada em nosso estudo.

Destarte, os achados desse estudo sugerem que, no caso dos agachamentos estudados, quando buscamos mecanoinduzir estímulos com o objetivo de propiciar respostas mioelétricas mais intensas, o exercício $\mathrm{AF}$ apresenta-se mais eficaz do que o $\mathrm{AP}$, principalmente para o músculo glúteo máximo, que respondeu com mais intensidade à modificação da posição de membros inferiores (FIGURA 2). No entanto, como a amostra foi pequena e a sobrecarga utilizada foi relativada a partir da massa corporal individual, é necessário cautela na inferência irrestrita dos resultados, principalmente quando da utilização de valores de resistência mais expressivos que os aqui adotados.

Mesmo que os achados sejam confirmados em estudos futuros, é de fundamental importância a observação de certos aspectos quando da prescrição tanto do AF quanto do AP, principalmente para indivíduos com histórico de diferentes tipos de lesões na articulação do joelho, tendo em vista o provável aumento do estresse patelo-femoral resultante da flexão do joelho entre $60^{\circ}$ e $90^{\circ}$. Nesse sentido recomenda-se que, antes de proceder à prescrição, que seja realizada anamnese e exame físico de modo a assegurar que o executante não apresente dor patelo-femoral, assim como fatores de risco para o desenvolvimento da mesma (EsCAMILLA et al., 2008; Leporace, Pereira, Carmo, Silva, Cabral, Silva Filho, Pasqualini \& Batista, 2010).

Quanto a sua utilização na reabilitação do ligamento cruzado anterior, EsCAMILLA, ZHENG, Macleod, Imamura, Edwards, Hreljac, Fleisig, Wilk, Moorman, Paulos e Andrews (2010) sugerem que ambos os exercícios podem ser prescritos durante todas as fases, mesmo após procedimentos cirúrgicos. Por outro lado, para o ligamento cruzado posterior, não se recomenda a prescrição do AF nas fases iniciais de reabilitação devido à alta tensão à qual esse ligamento é submetido entre $40^{\circ}$ e $90^{\circ}$ de flexão do joelho (EsCAmilla et al., 2010).

Sugere-se para estudos futuros a realização de pesquisas com modelos experimentais semelhantes, no entanto, que utilizem uma amostra maior e utilizem sobrecarga relativa à $1 \mathrm{RM}$. Como limitações desse estudo, destacam-se o baixo $\mathrm{N}$ amostral, o que recomenda prudência quando da generalização dos achados, e o baixo percentual de carga utilizada em relação à massa corporal, apesar disto não ter impedido a maior estimulação das musculaturas testadas. Além disso, uma limitação inata à técnica de eletromiografia foi à possível modificação no posicionamento dos eletrodos das musculaturas testadas, devido à diferente posição angular dos membros inferiores em cada exercício.

Em conclusão, os sujeitos testados manifestaram maior ativação mioelétrica do glúteo máximo e bíceps femoral no exercício de agachamento com passada à frente em comparação aos valores obtidos no agachamento paralelo para uma mesma carga absoluta. Por conseguinte, quando o objetivo da prescrição é estimular mais esses músculos, os achados do presente estudo sugerem a preconização do agachamento com passada à frente ao agachamento paralelo. 


\begin{abstract}
Comparison of myoelectric activity of gluteus maximus and biceps femoris between parallel and lunge squat

The purpose of this study was to compare the EMG of the gluteus maximus and biceps femoris between the lunge and the parallel squat. Seven subjects, with experience in strength training, performed eight repetitions of the parallel squat (PS) and the lunge (LU) with an overload corresponding to $50 \%$ of body mass. The EMG of the gluteus maximus and biceps femoris was captured, filtered by a forth order Butterworth filter $(20-400 \mathrm{~Hz})$ and calculated RMS values. The Wilcoxon Ranked test was used to compare the normalized EMG of each muscle between the two exercises. Both the biceps femoris $(p=0.041)$ and the gluteus maximus $(p=0.0059)$ showed increased activation in LU compared to the PS. Despite the moderate activation in both exercises, ranging from $25 \%$ to $45 \%$, the myoelectric response of the analyzed muscles was higher, for the participants, in the lunge exercise.
\end{abstract}

UnITERMS: Electromyography; Resistance training; Hip.

\title{
Resumen
}

Comparación de la activación mioeléctrica de los glúteos y bíceps femoral entre las sentadillas con los pies paralelos y los pies uno frente al otro

El objetivo de este estudio fue comparar la actividad mioeléctrica (EMG) del músculo glúteo mayor y el bíceps femoral entre la sentadilla con los pies uno frente al otro y los pies paralelos. Siete sujetos realizaron ocho repeticiones de la sentadilla paralela (AP) y con los pies uno frente al otro (FA) desplazando una masa igual a 50\% del peso corporal. El EMG del músculo glúteo mayor y el bíceps femoral fueron capturados, filtrada por un filtro de banda de $4{ }^{\circ}$ orden Butterworth $(20-400 \mathrm{~Hz})$ y los valores RMS obtenidos. Se utilizó el test de Wilcoxon para comparar la EMG normalizada entre los dos ejercicios. Tanto el bíceps femoral $(p=0,041)$ y el glúteo mayor $(p=0,0059)$ mostraron una mayor activación en la FA (30,9\% para lo glúteo y 52\% para lo bíceps) en comparación con el AP $(21,2 \%$ para lo glúteo y $24,7 \%$ para lo bíceps). Aunque la activación moderada durante la ejecución de los ejercicios fue moderado, situándose en torno a un 25\% a 45\%, la respuesta fue mayor en el FA para los participantes del estudio.

Palabras Clave: Electromiografia; Entrenamiento de resistencia; Cadera.

\section{Referências}

CATERISANO, A.; MOSS, R.E.; PELLINGER, T.K.; WOODRUFF, K.; LEWIS, V.C.; BOOTH, W.; KHADRA, T. The effect of back squat depth on the EMG activity of 4 superficial hip and thigh muscles. Journal of Strength and Conditioning Research, Champaign, v.16, p.428-32, 2002.

CHAGAS, M.H.; BARBOSA, J.R.M.; LIMA, F.V. Comparação do número máximo de repetições realizadas a 40 e $80 \%$ de uma repetição máxima em dois diferentes exercícios na musculação entre os gêneros masculino e feminino. Revista Brasileira de Educação Física e Esporte, São Paulo, v.19, n.1, p.5-12, 2005.

CRAM, J.; KASMAN, G.; HOLTZ, J. Introduction to surface electromyography. Gaithersburg: Aspen, 1998.

DA SILVA, E.M.; BRENTANO, M.A.; CADORE, E.L.; DE ALMEIDA, A.P.; KRUEL, L.F. Analysis of muscle activation during different leg press exercises at submaximum effort levels. Journal of Strength and Conditioning Research, Champaign, v.22, p.1059-65, 2008.

DE LUCA, C. The use of electromyography in biomechanics. Journal of Applied Biomechanics, Champaign, v.13, p.135-63, 1997. DWYER, M.K.; BOUDREAU, S.N.; MATTACOLA, C.G.; UHL, T.L.; LATTERMAN, C. Comparison of lower extremity kinematics and hip muscle activation during rehabilitation tasks between sexes. Journal of Athletic Training, Dallas, v.45, n.2, p.181-90, 2010. 
ESCAMILLA, R.F.; FLEISIG, G.S.; ZHENG, N.; LANDER, J.E.; BARRENTINE, S.W.; ANDREW, J.R.; BERGEMANN; B.W.; MOORMAN, C.T. Effects of technique variations on knee biomechanics during the squat and leg press. Medicine and Science in Sports Exercise, Madison, v.33, n.9, p.1552-66, 2001.

ESCAMILLA, R.F.; ZHENG, N.; MacLEOD, T.D.; EDWARDS, W.B.; HRELJAC, A.; FLEISIG, G.S.; WILK, K.E.; MOORMAN, C.T.; IMAMURA, R.; ANDREWS, J.R. Patellofemoral joint force and stress between a short- and longstep forward lunge. Journal of Ortophaedic and Sports Physical Therapy, Alexandria, v.38, n.11, p.681-90, 2008. ESCAMILLA, R.F.; ZHENG, N.; MacLEOD, T.D.; IMAMURA, R.; EDWARDS, W.B.; HRELJAC, A.; FLEISIG, G.S.; WILK, K.E.; MOORMAN, C.T., PAULOS, L.; ANDREWS, JR. Cruciate ligament tensile forces during the forward and side lunge. Clinical Biomechanics, Bristol, v.25, n.3, p.213-21, 2010.

FLECK, S.J. Periodized strength training: a critical review. Journal of Strength and Conditioning Research, Champaign, v.13, n.1, p.82-9, 1999.

FLECK, S.J.; KRAEMER, W.J. Fundamentos do treinamento de força muscular. São Paulo: Artes Médicas Sul, 1999. LEPORACE, G.; PEREIRA, G.R.; CARMO, R.C.R.; SILVA, A.C.; CABRAL, R.P.; SILVA FILHO, N.; PASQUALINE, H.E.C.; BATISTA, L.A. Especificidade da atividade mioelétrica no agachamento excêntrico declinado $25^{\circ}$ e no agachamento padrão com diferentes sobrecargas. Revista Brasileira de Medicina do Esporte, São Paulo, v.16, n.3, p.205-9, 2010. McCAW, S.; MELROSE, D. Stance width and bar load effects on leg muscle activity during the parallel squat. Medicine and Science in Sports Exercise, Madison, v.31, n.3, p.426-36, 1999.

PEREIRA, G.R.; LEPORACE, G.; CHAGAS, D.V.; FURTADO, L.F.L.; PRAXEDES, J.; BATISTA, L.A. Influence of hip external rotation on hip adductor and rectus femoris myoelectric activity during a dynamic parallel squat. Journal of Strength and Conditioning Research, Champaign, v.24, n.10, p.2749-54, 2010.

ENDEREÇO Gustavo Leporace Laboratório de Biomecânica e Comportamento Motor Universidade do Estado do Rio de J aneiro R. São Francisco Xavier, 524 - 8 andar - Ginásio Esportivo 20550-900 - Rio de Janeiro - RJ - BRASIL e-mail: gustavo.leporace@brasilsaude.org.br
Recebido para publicação: 17/ 05/ 2011

Revisado: 08/05/2012

Aceito: 25/ 05/ 2012 